\title{
Sonographic Evaluation of \\ Lower Extremity Interosseous Membrane Injuries
}

\author{
Retrospective Review in 3 Patients
}

\author{
$N$. Jarrod Durkee, $M D$, Jon A. Jacobson, $M D$, \\ David A. Jamadar, MBBS, FRCR, FRCS, John E. Femino, MD, \\ Madhav A. Karunakar, MD, Curtis W. Hayes, MD
}

\begin{abstract}
Objective. To describe the sonographic findings of lower extremity interosseous membrane disruption with computed tomographic and surgical correlation. Methods. Three patients with sonographic evaluation of the lower extremity interosseous membrane were identified through the clinical experience of 1 author over a 5-year period. Sonographic images of the symptomatic and unaffected lower extremities were retrospectively characterized and correlated with computed tomographic and surgical findings by 2 fellowship-trained musculoskeletal radiologists. Results. The normal interosseous membrane was identified in the asymptomatic lower extremities in all 3 patients, which appeared as a thin, hyperechoic (nearly equal to bone cortex) line, continuous from the tibia to the fibula. The abnormal interosseous membrane in all 3 cases appeared abnormally hypoechoic, poorly defined, and discontinuous at the tibia. A proximal fibular fracture was shown on sonography in 1 of 3 symptomatic lower extremities with radiographic evidence of a fracture. The sonographic findings correlated with the computed tomographic images. Distal tibiofibular syndesmosis injuries were confirmed and treated at surgery in 2 patients. Conclusions. Sonography can show both normal and injured interosseous membranes of the lower extremity, as well as associated proximal fibular fractures. Key words: ankle injuries; extremities; ligaments; soft tissues; sonography.
\end{abstract}

Abbreviations

$\mathrm{CT}$, computed tomography; IOM, interosseous membrane

Received April 19, 2003, from the Departments of Radiology (N.J.D., J.A.J., D.A.J., C.W.H.) and Orthopaedic Surgery (J.E.F., M.A.K.), University of Michigan Medical Center, Ann Arbor, Michigan USA. Revision requested May 20, 2003. Revised manuscript accepted for publication May 27, 2003. Address correspondence and reprint requests to Jon A. Jacobson, MD, Department of Radiology, University of Michigan Medical Center, 1500 E Medical Center Dr, TC-2910G, Ann Arbor, MI 481090326 USA.

E-mail: jjacobsn@umich.edu.

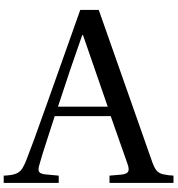

lthough common, ankle injuries vary considerably in severity and occur at all levels of athletic participation. ${ }^{1}$ Lateral ankle sprains are more common than high or syndesmotic ankle sprains; however, the latter often have longer recovery periods and are difficult to diagnose. ${ }^{1}$

The distal tibiofibular joint is a syndesmotic articulation stabilized by the anterior inferior tibiofibular ligament, the posterior inferior tibiofibular ligament, and the interosseous ligament (Fig. 1). ${ }^{1}$ Some consider a fourth structure, the transverse tibiofibular ligament, as the deep component of the posterior inferior tibiofibular ligament, whereas others consider this a discrete structure. ${ }^{1}$ The interosseous membrane (IOM) is a fibrous sheet 
between the tibia and fibula comprising obliquely oriented fibers, which thickens distally to become the interosseous ligament. The IOM has an important role in the transfer of forces to the fibula during weight bearing and, together with the other structures of the distal tibiofibular joint, is important in stabilization. ${ }^{1,2}$

The prevalence of IOM and syndesmosis injuries associated with ankle trauma has been described as $1 \%$ to $10 \% .^{3}$ Although the IOM is not directly seen on plain radiographs, widening of

Figure 1. Three-dimensional CT surface rendering of the lower extremity showing the IOM (blue), anterior inferior tibiofibular ligament (red), and posterior inferior and transverse tibiofibular ligaments (green).

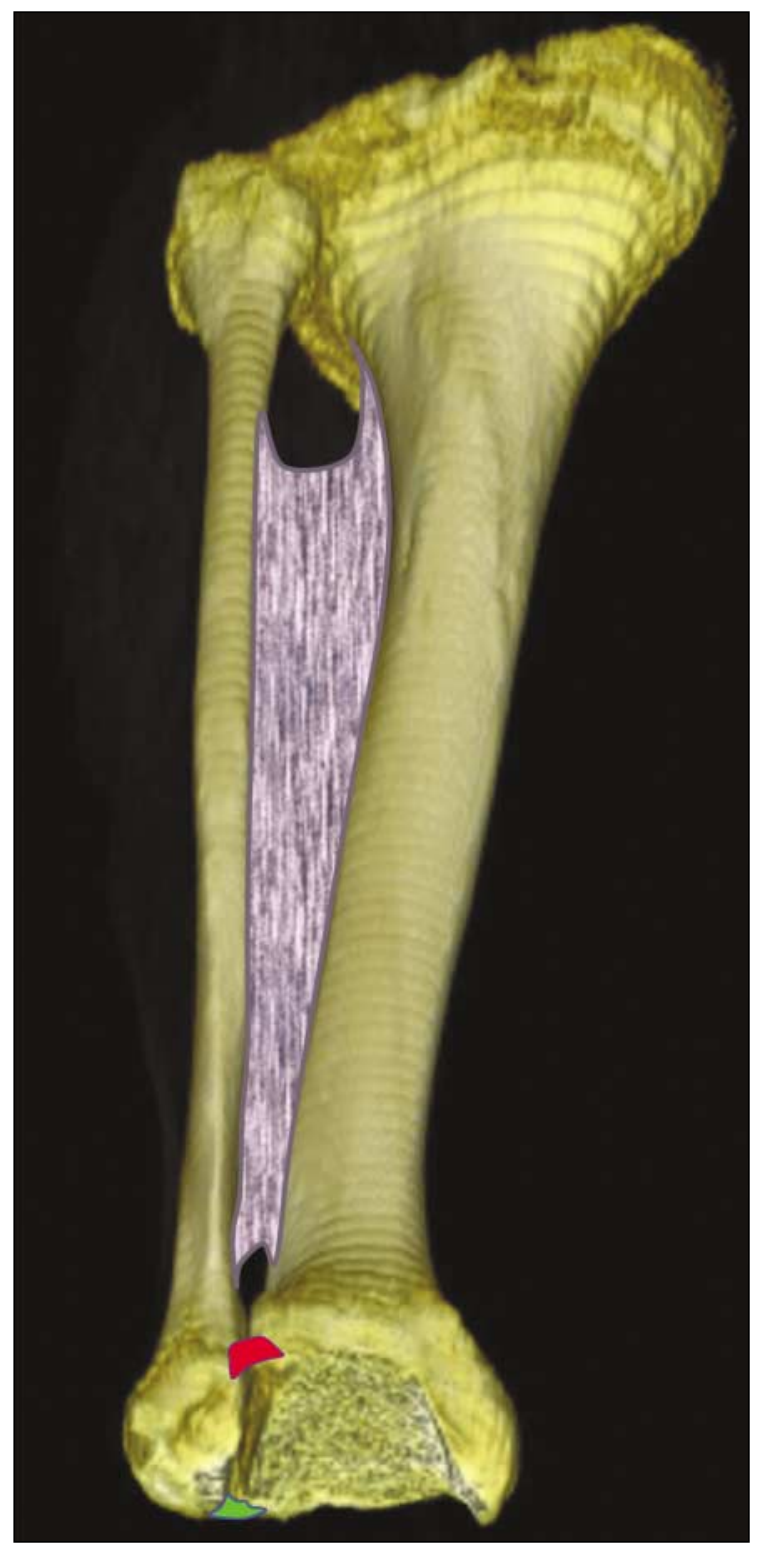

the distal tibiofibular syndesmosis indicates a ligament and possible IOM injury. However, not all syndesmotic injuries are associated with radiographic findings. ${ }^{1}$ Magnetic resonance imaging has been used to evaluate distal tibiofibular syndesmotic injuries. ${ }^{4-6}$ Although computed tomography (CT) is used primarily to investigate and to characterize osseous injuries, proper window and level settings can reveal tendon and ligament abnormalities of the foot and ankle as well. ${ }^{7}$ Sonography has been used in the evaluation of ankle tendons and ligaments. ${ }^{8-10}$ To our knowledge, there are only a few reports of sonography in evaluation of the IOM of the forearm ${ }^{11-13}$ and lower extremity ${ }^{2}$ and none in the radiologic literature. The purpose of this report is to describe the sonographic findings of lower extremity IOM disruption with CT and surgical correlation.

\section{Materials and Methods}

Permission to complete the study was granted by our Institutional Review Board. Three patients who underwent sonographic evaluation of the lower extremity IOM were identified through the clinical experience of 1 author over a 5-year period. Sonographic examinations were completed by 1 fellowship-trained musculoskeletal radiologist using $7-$ to $12-\mathrm{MHz}$ linear probes (HDI 3000 and HDI 5000; Philips Medical Systems, Bothell, WA). Liberal transmission gel was used in place of a standoff pad. To image the IOM, sonographic scanning was completed in an axial plane between the tibia and fibula from an anterior approach. The transducer sound beam was kept perpendicular to the expected plane of the IOM to avoid artifactual hypoechogenicity. The clinical indications for each sonographic examination varied but included evaluation of tendons, ligaments, and the IOM. All patients who underwent CT were included in the study. Sonography was performed without knowledge of the CT results. The group consisted of 3 male patients (mean age, 28 years; range, 19-36 years), each of whom had a sports-related twisting injury to the ankle.

The sonographic and CT images were retrospectively reviewed by 2 fellowship-trained musculoskeletal radiologists experienced in musculoskeletal sonography, with opinions rendered by consensus. All sonographic and CT images were collectively reviewed with knowledge of surgical outcomes when available. First, 
the IOM from the asymptomatic contralateral side was characterized on sonographic and CT images (when available). Then the IOM from the symptomatic extremity was compared with the contralateral asymptomatic IOM and characterized. Sonographic data included echogenicity of the IOM and the location and description of abnormal findings, such as abnormal echogenicity and the location of IOM disruption. The retrospective results were compared with routine radiography reports and surgical findings. Prospective sonographic reports were also reviewed for information about associated abnormalities.

\section{Results}

In the 3 patients, the asymptomatic lower extremity was imaged by sonography in all 3 and by CT in 1. With sonography, the normal lower extremity IOM was clearly visualized as a thin, hyperechoic structure (nearly equal to bone cortex) extending continuously between the tibia and fibula between the anterior and deep posterior compartments of the lower leg (Figs. 2A and $3 \mathrm{~A})$. Evaluation was focused over the mid and lower regions of the tibia and fibula. With CT, the normal IOM appeared as a thin, continuous membrane, equal to muscle attenuation in a similar location (Fig. 2B).

In each of the 3 patients, the symptomatic lower extremity was imaged with sonography and CT. At sonography, a normal IOM was not visualized. Instead, the abnormal IOM was hypoechoic and poorly defined. In addition, there was focal discontinuity of the IOM at the tibia consistent with complete disruption (Figs. 2C and 3B). The sonographic appearance of the abnormal IOM was similar in all 3 patients. On CT, the abnormal IOM in 2 of the 3 patients was poorly defined, with decreased attenuation, and discontinuous at the tibia; in addition, 1 of these 2 patients had fluid attenuation around the tibia, presumably from hematoma (Fig. 2D), and there was thickening of the IOM at the fibula in the other patient. In the third patient, the IOM was not visualized (Fig. 3C).

Additional soft tissue abnormalities were reported at sonography. They included tears of the anterior inferior tibiofibular ligament in all 3 patients and deltoid ligament disruption in 1. Prospective sonographic results with regard to the integrity of the IOM were correct in each case.
In all 3 cases, routine radiographs reported fractures of the fibula (proximal third of the fibula in 2 patients and middle third of the fibula in 1). In 1 patient, the proximal and mid thirds of the fibula were evaluated with sonography, on which the fracture was shown as cortical discontinuity and a step-off of the hyperechoic bone cortex (Fig. 3D). All patients had imaging within 1 week of their injuries. Two of the 3 underwent surgery, which showed complete syndesmotic disruption, and the distal tibiofibular syndesmosis was stabilized with screws. The third patient, who did not undergo surgery, was lost to follow-up.

\section{Discussion}

At sonography, the normal IOM appeared as a thin, hyperechoic (nearly equal to bone cortex), continuous structure, which extended between the tibia and fibula (Figs. 2A and 3A). In contrast, the disrupted IOM was poorly defined, abnormally hypoechoic, and focally discontinuous at the tibia in all 3 patients (Figs. 2C and 3B). The imaging findings on CT correlated with the sonographic findings, in which the normal IOM was a thin, continuous membrane, equal in attenuation to muscle, located between the tibia and fibula (Fig. 2B). The abnormal IOM was either not visualized or focally discontinuous with decreased attenuation on CT (Figs. 2D and 3C).

Distal tibiofibular syndesmotic injuries, either complete or incomplete, can occur by a variety of injury mechanisms, which include supination and external rotation, supination and adduction, pronation and external rotation, and pronation and abduction. ${ }^{1,14,15}$ Many are associated with fractures of the medial malleolus, posterior tubercle of the tibia, and fibula. ${ }^{14,15}$ Christodoulou et $\mathrm{al}^{2}$ reported rupture of the IOM in $100 \%$ of Weber type C fractures and $23 \%$ of Weber type B fractures. When associated with a fracture of the proximal third of the fibula (Maisonneuve fracture), the interosseous ligament is usually torn. ${ }^{14}$ However, the extent of IOM injuries in this setting is not known because tears may involve only its distal aspect, ${ }^{5}$ can extend proximal to a high fibular fracture, ${ }^{2}$ or may be absent. ${ }^{4,14}$ Interosseous membrane tears can occur in all types of ankle fractures ${ }^{2}$; therefore, the presence or extent of IOM tears cannot be predicted from radiographic findings alone. 


\section{Sonography of Lower Extremity Interosseous Membrane Injuries}

Figure 2. Interosseous membrane disruption in a 29-year-old man. A, Transverse sonogram of the asymptomatic lower extremity at the level of the mid tibia showing a normal thin, hyperechoic, continuous IOM (arrowhead) between the tibia (T) and fibula (F; the apparent discontinuity at the fibula is technical). B, Corresponding transverse non-contrast-enhanced CT image showing the intact IOM (arrowhead). C, Transverse sonogram of the symptomatic lower extremity showing hypoechoic discontinuity (arrow) of the IOM (arrowhead) near the tibia. D, Transverse CT image showing discontinuity (arrow) of the IOM (arrowhead) and hematoma, which surrounds the tibia.

A

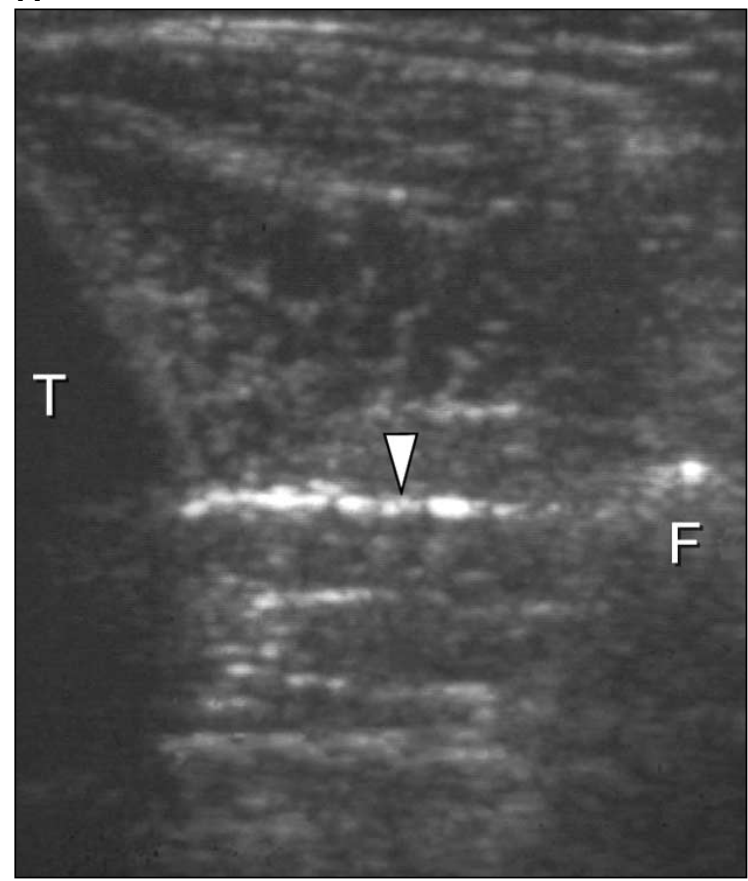

C

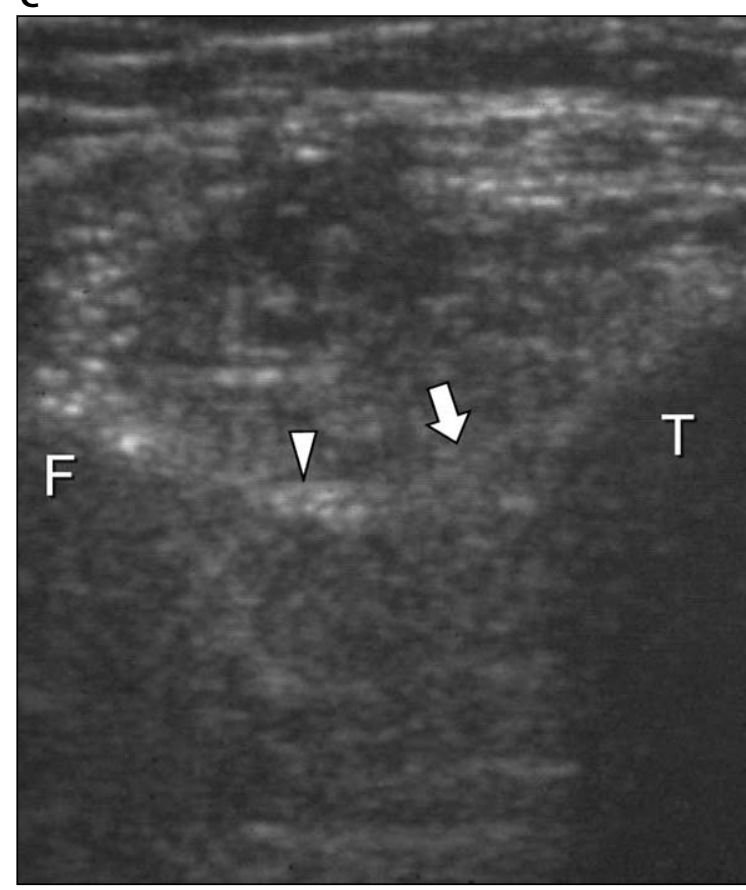

B

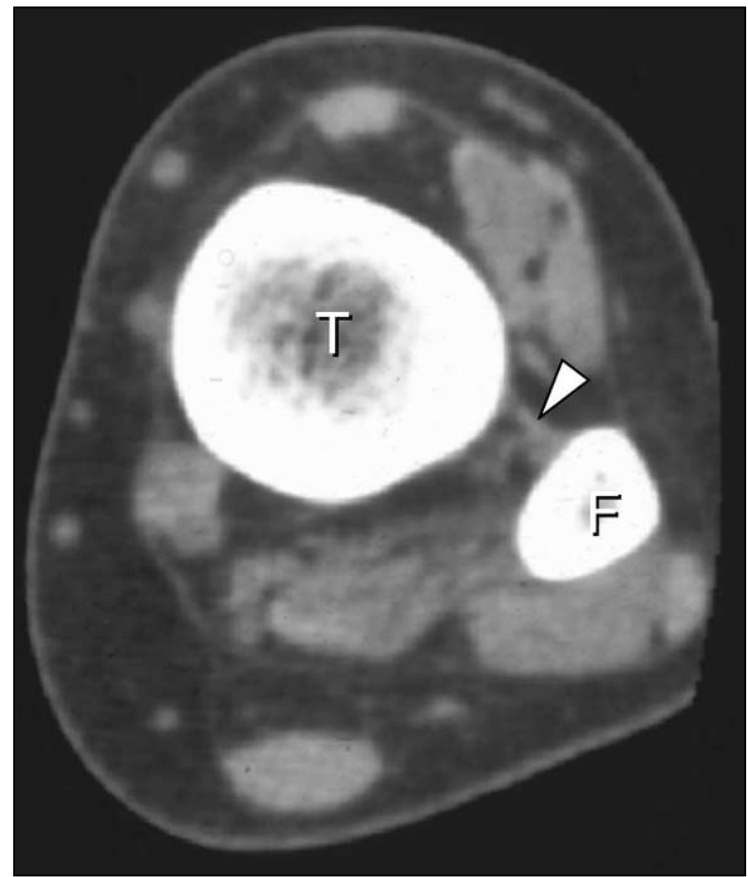

D

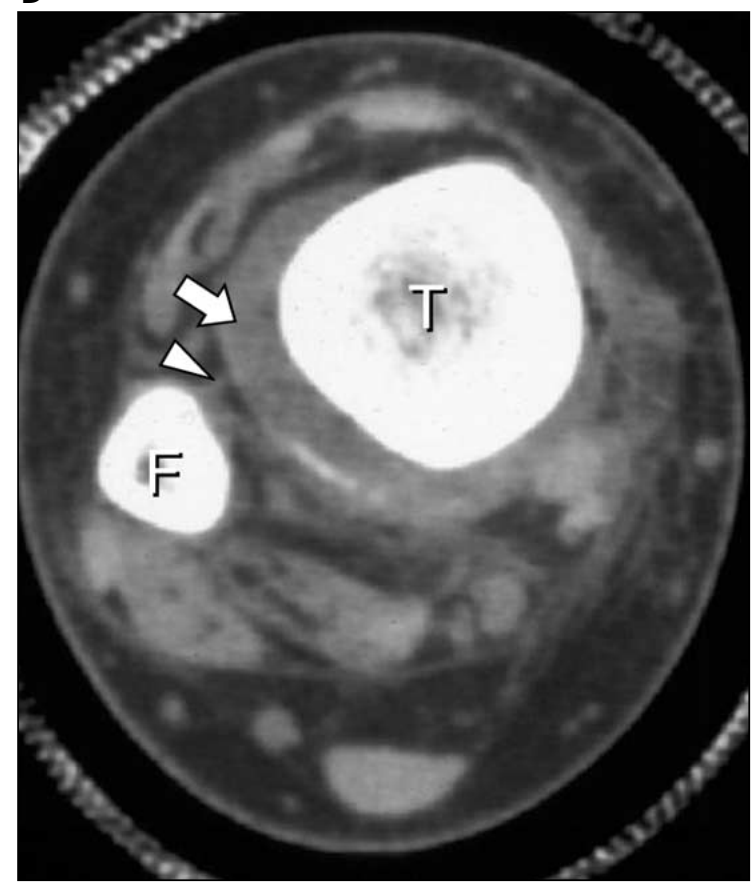


Figure 3. Maisonneuve fracture and IOM disruption in a 36-year-old man. A, Transverse sonogram of the asymptomatic lower extremity at the level of the mid tibia showing a normal thin, hyperechoic, continuous IOM (arrowhead) between the tibia (T) and fibula (F). B, Transverse sonogram of the symptomatic lower extremity showing hypoechoic discontinuity (arrow) of the IOM (arrowhead) near the tibia. C, Transverse CT image, which does not show the IOM discontinuity (arrow). D, Sonogram longitudinal to the proximal fibula of the symptomatic lower extremity showing discontinuity and a step-off deformity (arrow) of the fibula cortex, which represents a proximal fibular fracture.

A

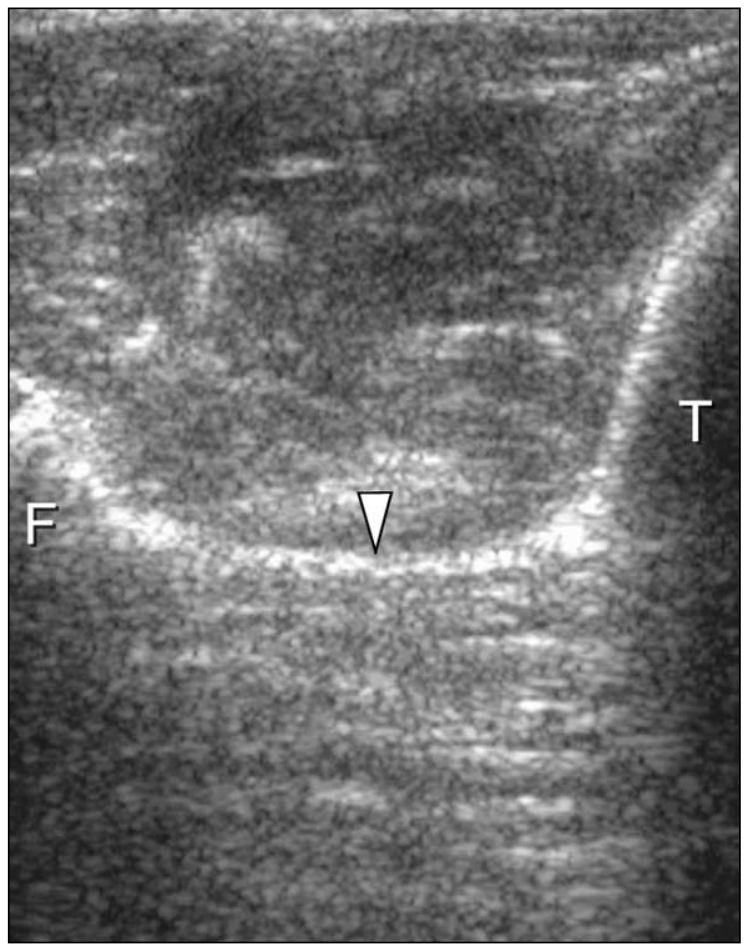

C

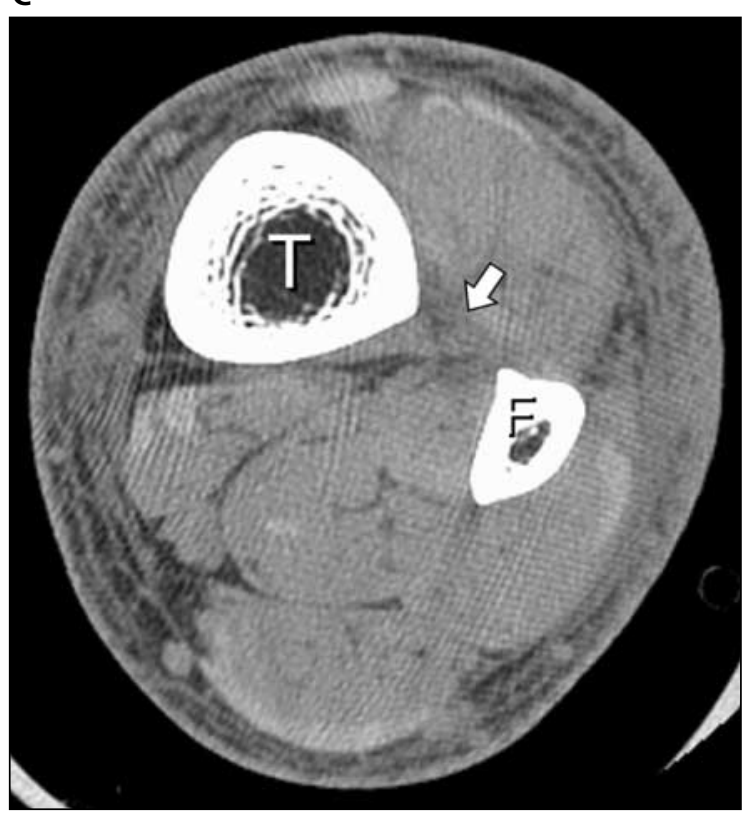

B

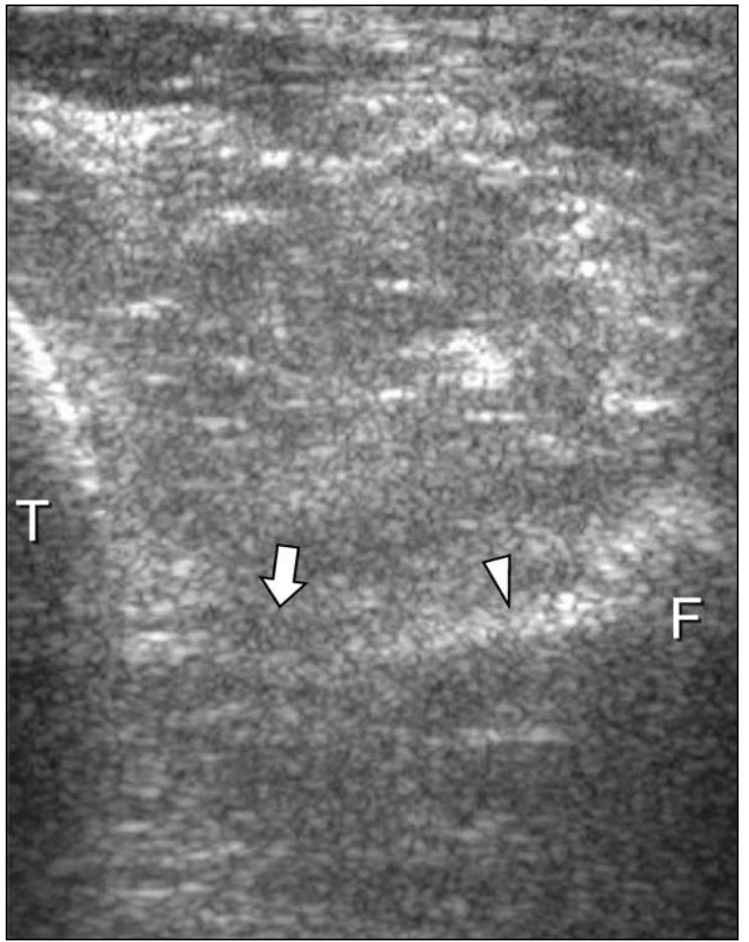

D

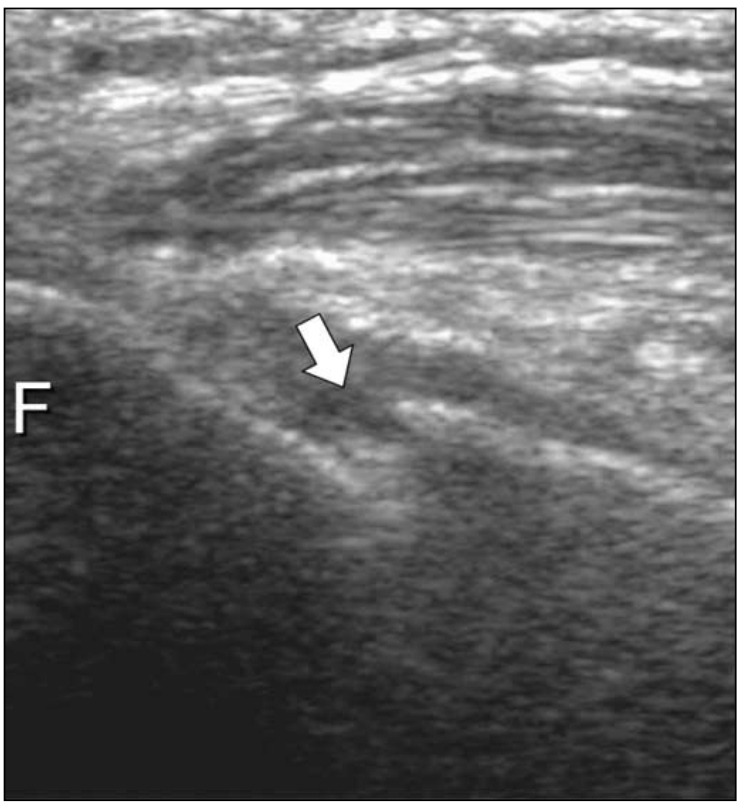


At radiography, a distal tibiofibular ligament injury is present when there is widening of the distal syndesmotic articulation. In adults, the normal distal tibiofibular syndesmosis measures less than $6 \mathrm{~mm}$ at a level $1 \mathrm{~cm}$ above the tibial plafond on anteroposterior or mortise ankle views, with tibiofibular overlap of greater than $6 \mathrm{~mm}$ and $1 \mathrm{~mm}$ on anteroposterior and mortise views, respectively. ${ }^{16}$ Magnetic resonance imaging has been used to evaluate the distal tibiofibular syndesmosis. ${ }^{4-6}$ An intact or thinned ligament or an IOM surrounded by high-signal edema or hemorrhage on transverse relaxation time-weighted sequences is consistent with a partial-thickness tear. ${ }^{4-6}$ A complete ligament tear is characterized by ligament or IOM disruption or nonvisualization..$^{4-6}$ Sonography can be used effectively to evaluate the acutely traumatized ankle, such as in the evaluation of an ankle ligament injury. ${ }^{8-10}$ Our results show the ability of sonography to facilitate the diagnosis of complete IOM disruption and proximal fibular fractures (Fig. 3D). Sonography should be considered for evaluation of a ligament and IOM injury when ankle radiographic findings are normal and clinical findings suggest a high ankle sprain. In addition, sonography can be used to evaluate the extent of IOM disruption when ankle mortise widening is present on radiography. If the extent of IOM disruption is precisely determined by sonography, the amount of exploration and the extent of the surgical incision can be minimized. ${ }^{2}$ Therefore, sonography may provide a diagnosis to enable prompt and appropriate treatment.

Distal tibiofibular syndesmotic injuries are associated with tears of the anterior inferior tibiofibular, posterior inferior tibiofibular, interosseous, deltoid, anterior talofibular, and calcaneofibular ligaments. ${ }^{2,4-6}$ When one of these ligaments is found to be abnormal on sonography, it is important to consider a high ankle sprain and a syndesmotic injury. For example, identification of an anterior talofibular or deltoid ligament tear warrants examination of the anterior inferior tibiofibular ligament, IOM, and proximal fibula to exclude syndesmotic disruption.

Limitations of this study include its retrospective nature, the small number of patients, and CT imaging of only 1 normal IOM. Further investigation in a larger patient population would determine whether these preliminary results could be applied to the general population.
In summary, sonography can show an abnormal lower extremity IOM as hypoechoic, poorly defined, and discontinuous. In addition to sonographic evaluation of the ankle ligaments after trauma, evaluation of the IOM and proximal fibula for coexisting injury also should be included. Sonography can be considered for evaluation of soft tissue injury in the lower extremity instead of more expensive CT or magnetic resonance imaging.

\section{References}

1. Norkus SA, Floyd RT. The anatomy and mechanisms of syndesmotic ankle sprains. J Athl Train 2001; 36:68-73.

2. Christodoulou G, Korovessis P, Giarmenitis S, Dimopoulos P, Sdougos G. The use of sonography for evaluation of the integrity and healing process of the tibiofibular interosseous membrane in ankle fractures. J Orthop Trauma 1995; 9:98-106.

3. Bassewitz HL, Shapiro MS. Persistent pain after ankle sprain: targeting the causes. Phys Sportsmed 1997; 25:59-68.

4. Morris JR, Lee J, Thordarson D, Terk MR, Brustein M. Magnetic resonance imaging of acute Maisonneuve fractures. Foot Ankle Int 1996; 17:259-263.

5. Manyi W, Guowei R, Shensong Y, Chunyan J. A sample of Chinese literature MRI diagnosis of interosseous membrane injury in Maisonneuve fractures of the fibula. Injury 2000; 31:107-110.

6. Vogl TJ, Hochmuth K, Diebold T, et al. Magnetic resonance imaging in the diagnosis of acute injured distal tibiofibular syndesmosis. Invest Radiol 1997; 32: 401-409.

7. Keyser CK, Gilula LA, Hardy DC, Adler S, Vannier M. Soft-tissue abnormalities of the foot and ankle: CT diagnosis. AJR Am J Roentgenol 1988; 150:845850.

8. Fessell DP, Vanderschueren GM, Jacobson JA, et al. US of the ankle: technique, anatomy, and diagnosis of pathologic conditions. Radiographics 1998; 18:325-340.

9. Milz P, Milz S, Putz R, Reiser M. $13 \mathrm{MHz}$ highfrequency sonography of the lateral ankle joint ligaments and the tibiofibular syndesmosis in anatomic specimens. J Ultrasound Med 1996; 15:277-284.

10. Campbell DG, Menz A, Isaacs J. Dynamic ankle ultrasonography: a new imaging technique for acute 
ankle ligament injuries. Am J Sport Med 1994; 22:855-858.

11. Failla JM, Jacobson JA, van Holsbeeck M. Ultrasound diagnosis and surgical pathology of the torn interosseous membrane in forearm fractures/dislocations. J Hand Surg [Am] 1999; 24:257-266.

12. Jaakkola Jl, Riggans DH, Lourie GM, Lang CJ, El Hassem B, Rosenthal SJ. Ultrasonography for evaluation of forearm interosseous membrane disruption in a cadaver model. J Hand Surg [Am] 2001; 26: 1053-1057.

13. Fester EW, Murray PM, Sanders TG, Ingari $J$, Leyendecker J, Leis HL. The efficacy of magnetic resonance imaging and ultrasound in detecting disruptions of the forearm interosseous membrane: a cadaver study. J Hand Surg [Am] 2002; 27:418-424.

14. Pankovich AM. Maisonneuve fracture of the fibula. J Bone Joint Surg Am 1976; 558:337-342.

15. Pankovich AM. Fractures of the fibula proximal to the distal tibiofibular syndesmosis. J Bone Joint Surg Am 1978; 60:221-229.

16. Bozic KJ, Jaramillo D, DiCanzio J, Zurakowski D, Kasser JR. Radiographic appearance of the normal distal tibiofibular syndesmosis in children. J Pediatr Orthop 1999; 19:14-21. 